Louisiana State University

LSU Digital Commons

6-1-2013

\title{
Trade-offs, geography, and limits to thermal adaptation in a tide pool copepod
}

Morgan W. Kelly

University of California, Davis

Richard K. Grosberg

University of California, Davis

Eric Sanford

University of California, Davis

Follow this and additional works at: https://digitalcommons.Isu.edu/biosci_pubs

\section{Recommended Citation}

Kelly, M., Grosberg, R., \& Sanford, E. (2013). Trade-offs, geography, and limits to thermal adaptation in a tide pool copepod. American Naturalist, 181 (6), 846-854. https://doi.org/10.1086/670336

This Article is brought to you for free and open access by the Department of Biological Sciences at LSU Digital Commons. It has been accepted for inclusion in Faculty Publications by an authorized administrator of LSU Digital Commons. For more information, please contact ir@lsu.edu. 
VOL. 181, NO. 6 THE AMERICAN NATURALIST JUNE 2013

Note

\title{
Trade-Offs, Geography, and Limits to Thermal Adaptation in a Tide Pool Copepod
}

\author{
Morgan W. Kelly, ${ }^{1,2, *}$ Richard K. Grosberg, ${ }^{1}$ and Eric Sanford ${ }^{1,2}$ \\ 1. Department of Evolution and Ecology, University of California, Davis, California 95616; 2. Bodega Marine Laboratory, University of \\ California, Davis, Bodega Bay, California 94923
}

Submitted October 16, 2012; Accepted February 13, 2013; Electronically published April 26, 2013

Online enhancements: appendix figures. Dryad data: http://dx.doi.org/10.5061/dryad.g89m0.

\begin{abstract}
Antagonistic correlations among traits may slow the rate of adaptation to a changing environment. The tide pool copepod Tigriopus californicus is locally adapted to temperature, but within populations, the response to selection for increased heat tolerance plateaus rapidly, suggesting either limited variation within populations or costs of increased tolerance. To measure possible costs of thermal tolerance, we selected for increased upper lethal limits for 10 generations in 22 lines of $T$. californicus from six populations. Then, for each line, we measured six fitness-related traits. Selected lines showed an overall increase in male and female body sizes, fecundity, and starvation resistance, suggesting a small benefit from (rather than costs of) increased tolerance. The effect of selection on correlated traits also varied significantly by population for five traits, indicating that the genetic basis for the selection response differed among populations. Our results suggest that adaptation was limited by the presence of variation within isolated populations rather than by costs of increased tolerance.
\end{abstract}

Keywords: adaptation, climate change, constraint, $\mathbf{G}$ matrix, genetic correlation, heat tolerance.

\section{Introduction}

Trade-offs occur whenever competing demands on an organism prevent fitness from being maximized, and they can influence both the magnitude and the direction of evolution (Agrawal et al. 2010). Trade-offs may be generated by opposing selection on a single trait (Mauricio and Rausher 1997) or positive selection on two traits sharing a limiting resource (Worley and Barrett 2000). In either case, the direction of evolution depends on not just the additive variance for single traits but also the additive variance in the multivariate direction of selection, that is, the variance present in the traits under selection in a particular environment and the genetic correlations among these

* Corresponding author; e-mail: morgan.kelly@lifesci.ucsb.edu.

Am. Nat. 2013. Vol. 181, pp. 846-854. (C) 2013 by The University of Chicago. 0003-0147/2013/18106-54185\$15.00. All rights reserved.

DOI: $10.1086 / 670336$ traits (Lande and Arnold 1983; Blows and Hoffmann 2005; Agrawal and Stinchcombe 2009).

From this perspective, the problem of predicting how or whether organisms can adapt to increased stress during periods of environmental change is inherently a challenge of deciphering the interaction between a changing selective regime and the genetic variances and covariances of the traits under selection. Although physiological responses to environmental stress have received increased attention in recent years (see Chown and Gaston 2008), few studies explicitly consider the capacities of natural populations to evolve increased stress tolerance (but see Jump and Peñuelas 2005; Balanya et al. 2006; Franks et al. 2007; Hoffmann and Sgrò 2011; Franks and Hoffmann 2012; Lohbeck et al. 2012). Even fewer have considered trade-offs involved in stress adaptation (but see Etterson and Shaw 2001; Williams et al. 2012), limiting the ability to predict both the rate and correlated effects of evolutionary responses to environmental change.

Several lines of evidence suggest that the evolution of increased stress tolerance may be associated with costs (i.e., antagonistic selection on tolerance traits). Evolution of resistance to herbivores and insecticides often involves costs of reduced fecundity, growth, or survival in resistant individuals (Carriere et al. 1994; Mauricio and Rausher 1997; Janmaat and Myers 2005), and in laboratory selection experiments, limits to evolution are often reached due to negative pleiotropic effects of alleles under selection (Barton and Turelli 1989).

In natural settings, stress-tolerance trade-offs may be generated by negative correlations with other traits under selection. For example, Etterson and Shaw (2001) found that the predicted responses to selection on single traits related to drought tolerance were much greater than the predicted responses when correlations among those traits were taken into account. Nevertheless, it is also important to note that even strong correlations do not preclude rapid evolution (Conner et al. 2011). 
Whether they stem from antagonistic selection on single traits or genetic correlations among traits, trade-offs are notoriously difficult to measure for several reasons. First, nongenetic correlations among traits may accumulate due to environmental covariance in selection pressures (Stebbins 1950; Armbruster and Schwaegerle 1996). For example, species that experience selection for increased upper thermal limits may be less likely to experience selection for tolerance to freezing. Therefore, comparisons of heat and freezing tolerances among species may reveal a negative correlation between these two traits, even if there is no genetic association between them (e.g., Hoffmann et al. 2002 vs. Bubliy and Loeschcke 2005). Second, variation in resource levels among individuals will tend to mask underlying functional trade-offs among traits, because individuals that have acquired more resources tend to have larger values for all traits (Charlesworth 1990). Finally, even bivariate trait correlations measured via family breeding designs may fail to predict genetic correlations when families differ in their ability to acquire limiting resources (Houle 1991). Consequently, where feasible, selection experiments are among the most effective ways to characterize trade-offs generated by correlations among traits (Brakefield 2003; Fry 2003).

A final complication in the measurement of trade-offs is the instability of trait correlations over time and space (Turelli 1988; Jones et al. 2003; Doroszuk et al. 2008), meaning that measurements made on a single population or in a single environment may not generally predict responses in other populations or in other environments. The G matrix, which represents the genetic variance and covariance present in a set of traits, may differ among not only closely related species but also geographically separated populations of the same species (Cohan and Hoffmann 1986, 1989; Roff et al. 2004). If multiple loci control each of two traits, some alleles may have positive effects on both traits $(+/+)$, while others have positive effects on one and negative effects on the other $(+/-)$. Through sampling effects, two populations may be fixed for different numbers of $+/+$ or $+/-$ loci, leading to variation in the correlated response to selection on either trait (Gromko 1995). Indeed, empirical data suggest that bottlenecks and drift may lead to persistent changes in the $\mathbf{G}$ matrix (Shaw et al. 1995; Whitlock et al. 2002).

Here, we explore trade-offs that potentially influence the evolution of increased thermal tolerance in multiple geographically separated populations of the tide pool copepod Tigriopus californicus and ask whether correlations among thermal tolerance and other traits differ across populations of this species. Tigriopus californicus ranges over $3,000 \mathrm{~km}$ of latitude from Baja California $\left(27^{\circ} \mathrm{N}\right)$ to southeast Alaska $\left(57^{\circ} \mathrm{N}\right)$ but is restricted to high intertidal and supralittoral rocky pools with low connectivity among populations (Burton and Lee 1994). Because these pools experience long periods of tidal emersion, this copepod must tolerate a broad range of diurnal and seasonal variations in temperature. The combination of low gene flow among populations and a broad latitudinal distribution increases the probability of adaptation to local conditions. Previously, we showed that T. californicus is locally adapted to temperature, with southern populations having up to $3.5^{\circ} \mathrm{C}$ greater thermal tolerance than copepods from cooler northern sites (Kelly et al. 2012). However, the capacity to evolve increased upper thermal limits within populations is extremely small in comparison to the differences among populations, with a $\mathrm{Q}_{\mathrm{ST}}$ (a measure of population subdivision for quantitative traits; Spitze 1993) for thermal tolerance of $>0.99$ and an apparent limit to the response to selection for increased tolerance after five generations in most populations (fig. 1; Kelly et al. 2012). There are two possible causes for the observed bounds on the selection response: (1) limited additive genetic variance for thermal tolerance within populations and (2) costs associated with increased thermal tolerance imposed by negative pleiotropic effects of alleles conferring increased tolerance (leading to stabilizing selection on these alleles). The observed limits to selection are unlikely to have been driven by drift in laboratory populations, which were kept at 40 breeding pairs per generation. Theory predicts that half-life of the response to artificial selection is $\sim 1.4 N_{\mathrm{e}}$ generations (Robertson 1960), and in practice, limits to selection in the laboratory are rarely observed after only five generations (reviewed in Weber and Diggins 1990, but see Hoffmann et al. 2003a).

Recent work on T. californicus suggests that there is a trade-off in thermal performance, with the competitive dominance of northern versus southern $T$. californicus shifting as a function of temperature (Willett 2010). However, trade-offs for performance at optimal temperatures will not necessarily be the same as trade-offs for increased upper lethal limits (Loeschcke and Krebs 1996; Hoffmann et al. 1997).

Trade-offs involved in thermal adaptation remain poorly understood (Huey and Kingsolver 1993; Loeschcke and Krebs 1996; Gilchrist et al. 1997; Hoffmann et al. 1997, 2003b; Mongold et al. 1999; Palaima and Spitze 2004; Bubliy and Loeschcke 2005; Williams et al. 2012), and relatively few studies have assessed geographic variation in the $\mathbf{G}$ matrix (Cohan and Hoffmann 1989; Cano et al. 2004; Roff et al. 2004). We used 22 lines of T. californicus collected from six populations and selected for increased thermal tolerance for 10 generations to address two questions: (1) Do fitness trade-offs limit the response to selection for increased thermal tolerance in T. californicus? and (2) Do the correlated responses to selection differ among populations? We found that four out of six fitness-related traits 




Figure 1: Sampling locations for Tigriopus californicus: Fogarty Creek (FC; $\left.44^{\circ} 50^{\prime} \mathrm{N}, 124^{\circ} 03^{\prime} \mathrm{W}\right)$ and Strawberry Hill $\left(\mathrm{SH} ; 44^{\circ} 15^{\prime} \mathrm{N}, 124^{\circ} 06^{\prime} \mathrm{W}\right)$, Oregon; Salt Point (SA; $\left.38^{\circ} 20^{\prime} \mathrm{N}, 123^{\circ} 33^{\prime} \mathrm{W}\right)$, Bodega Marine Reserve (BR; $\left.38^{\circ} 04^{\prime} \mathrm{N}, 123^{\circ} 19^{\prime} \mathrm{W}\right)$, Bird Rock $\left(\mathrm{BD} ; 32^{\circ} 49^{\prime} \mathrm{N}, 117^{\circ} 16^{\prime} \mathrm{W}\right)$, and Cabrillo Point $\left(\mathrm{CB} ; 32^{\circ} 04^{\prime} \mathrm{N}, 117^{\circ} 15^{\prime} \mathrm{W}\right)$, California. Mean thermal tolerance $\left(\mathrm{LT}_{50}\right) \pm \mathrm{SE}$ of adult male T. californicus from lines selected for 9-10 generations for increased thermal tolerance (light gray bars) and unselected controls (dark gray bars) from these six populations $(N=3-4$ selected and unselected lines per population). Bars for each population arranged vertically by latitude. Modified from Kelly et al. (2012).

showed a correlated response to selection on thermal tolerance, but we uncovered no evidence that evolution of increased tolerance in laboratory culture was limited by costs.

\section{Methods}

\section{Field Collection and Selection for Increased Thermal Tolerance}

Field collection, copepod culture, and laboratory selection experiments are described in detail in Kelly et al. (2012). Briefly, we established 22 laboratory cultures of Tigriopus californicus from six sites, ranging from Oregon to Southern California (fig. 1). At each site, we collected individuals from three to four tide pools. These pools included both the deepest/most shaded and the shallowest/most exposed pools we could find, encompassing the range of thermal conditions present at a site. We initiated one laboratory culture (line) for each pool with 50 gravid females per culture and maintained cultures at $19^{\circ} \mathrm{C}$ under $12 \mathrm{~L}: 12 \mathrm{D}$ conditions. We selected for increased thermal tolerance starting in the second laboratory-reared generation by exposing each of the 22 lines to 10 generations of mass selection. For each laboratory-reared culture, we exposed 100-400 mate-guarding pairs (males and virgin females) to $2 \mathrm{~h}$ of ramp-up from $20^{\circ} \mathrm{C}$ plus $1 \mathrm{~h}$ at the target tem- perature (the temperature that produced $40 \%-90 \%$ mortality in adult males). These same temperatures typically produced about $20 \%$ lower mortality in females, which have higher thermal tolerance than males. Data from temperature loggers in natural pools inhabited by $T$. californicus suggest that this is a realistic rate of temperature change during a tidal cycle (Kelly et al. 2012). We founded the subsequent generation in each culture using exactly 40 mate-guarding pairs selected randomly from the surviving individuals in each line. For each culture, we also maintained an unselected line under the same conditions, establishing each generation using 40 haphazardly selected mate-guarding pairs.

We gauged the response to selection on thermal tolerance in six traits important to overall fitness, measuring these traits in both selected lines and unselected controls between generations 8 and 10 of selection as described below. To account for limits to selection observed in the lab, fitness costs imposed by increased thermal tolerance would need to be exacted in the lab environment. We therefore measured clutch size, interclutch interval, and minimum generation time, all of which contribute to a population's intrinsic rate of increase. We also measured male and female body sizes, as body size is strongly tied to fecundity in female invertebrates (Shine 1988) and might be tied to success at competing for mates in males. 
Finally, we measured starvation resistance, which might have been tied to metabolic efficiency and, therefore, to fitness if there were some competition for food within cultures.

\section{Fecundity, Generation Time, and Interclutch Interval}

We placed mate-guarding pairs $(N=6-8$ per selected or unselected line) in 15-mL 6-well plates filled with filtered seawater and maintained them at $19^{\circ} \mathrm{C}$ under $12 \mathrm{~L}: 12 \mathrm{D}$ conditions. All adults and larvae in this experiment were fed ground spirulina fish food ad libitum. We recorded the day the female of each pair first became gravid and the dates of her first three broods. Each time a new brood hatched, the female was moved to a new well in the plate. Offspring from each brood were counted by individually pipetting larvae to a new dish. To calculate minimum generation time, the first brood for each female was transferred to a $50-\mathrm{mL}$ container and maintained at $19^{\circ} \mathrm{C}$ until the first daughter from that brood became gravid. We calculated mean interbrood interval for each female as the number of days between the hatching of the first and third broods divided by 2 . We estimated clutch size as the mean number of offspring in her first three broods.

\section{Starvation Resistance}

We estimated starvation resistance for each line as the mean number of days females survived without food. For each selected and unselected line, we placed 20-30 newly matured adult females into $50-\mathrm{mL}$ containers of filtered seawater. We assessed mortality by counting the number of females remaining every 5-7 days. Females were counted by individually pipetting them into new containers of filtered seawater. Throughout their lives prior to the experiment, females were fed ad libitum; throughout the experiment, cultures were maintained at $19^{\circ} \mathrm{C}$.

\section{Size}

We quantified adult size by photographing individual copepods and then measuring prosome and urosome lengths in Image $\mathrm{J}$ software (http://rsb.info.nih.gov/ij/), using straight lines drawn between standardized morphological landmarks (fig. A1, available online). We measured six adult females and six adult males for each selected line and unselected control. Body size was calculated as the sum of prosome and urosome lengths.

\section{Statistical Methods}

To investigate a correlated response to selection and assess whether such a response varied by population and line, we tested the fixed effects of selection on increased thermal tolerance, population of origin, and line (nested within population) for each of the six measured traits with separate ANOVAs using the statistical program R (R Development Core Team 2010). Female size was log transformed and interbrood interval and generation time were inverse transformed to achieve homoscedasticity of residuals. All other trait values were normally distributed with equal variance among treatments.

\section{Results}

Our previously published results indicated that five out of six populations responded to selection for increased thermal tolerance, with the maximum response being $0.53^{\circ} \mathrm{C}$ in the population from Bodega Marine Reserve, California (Kelly et al. 2012; fig. 1). Here, we found that selection for increased thermal tolerance produced an overall positive correlated response in four of six fitness-related traits (table 1; fig. 2). Lines selected for higher thermal tolerance evolved larger male and female body sizes, greater fecundity, and increased starvation resistance. The effects of selection varied significantly by population for five traits, including female size, clutch size, interclutch interval, generation time, and starvation resistance. However, variation in the effects of selection among lines within populations was greater than variation in the effects of selection on different populations, with about twice as much variation explained by the interaction between selection and line compared to the interaction between selection and population (table 1). There were no clear latitudinal trends for most traits, although the two southernmost populations (at Cabrillo Point and Bird Rock, CA) had smaller male and female body sizes than other populations. There was also no indication that trait values for selected lines in northern populations became more similar to trait values expressed by southern, more thermally tolerant populations.

\section{Discussion}

In this study, we sought to understand why the copepod Tigriopus californicus shows a limited response to selection for increased thermal tolerance within populations (Kelly et al. 2012). If the observed plateau in the selection response were due to balancing selection imposed by negative pleiotropic effects of alleles conferring increased tolerance, then we would predict that lines selected for increased tolerance would have lower fitness than unselected lines. We would also expect increased thermal tolerance to lead to changes in fitness-related traits if these traits shared limiting resources, for example, if energy used to manufacture increased heat shock proteins tended to divert energy away from egg production. However, after 
Table 1: Effects of selection for increased thermal tolerance on other traits for Tigriopus californicus from six geographically separated populations

\begin{tabular}{|c|c|c|c|c|c|c|}
\hline \multirow[b]{2}{*}{ Trait } & \multirow[b]{2}{*}{$\begin{array}{l}\text { Indirect } \\
\text { effect of } \\
\text { selection }\end{array}$} & \multicolumn{5}{|c|}{ Proportion of variance explained $\left(\eta^{2}\right)$} \\
\hline & & Selection & Population & $\begin{array}{c}\text { Line } \\
\text { (population) }\end{array}$ & $\begin{array}{l}\text { Selection } \times \\
\text { population }\end{array}$ & $\begin{array}{l}\text { Selection } \times \text { line } \\
\quad(\text { population })\end{array}$ \\
\hline Female size & Increase & $\begin{array}{c}.04^{* * *} \\
\left(F_{1,242}=30.6\right)\end{array}$ & $\begin{array}{c}.37^{* * *} \\
\left(F_{5,242}=63.4\right)\end{array}$ & $\begin{array}{c}.17^{* * *} \\
\left(F_{17,242}=8.9\right)\end{array}$ & $\begin{array}{c}.05^{* * *} \\
\left(F_{5,242}=9.06\right)\end{array}$ & $\begin{array}{c}.09^{* * *} \\
\left(F_{15,242}=5.4\right)\end{array}$ \\
\hline Male size & Increase & $\begin{array}{c}.09^{* * *} \\
\left(F_{1,169}=40.2\right)\end{array}$ & $\begin{array}{c}.18^{* * *} \\
\left(F_{5,169}=15.7\right)\end{array}$ & $\begin{array}{c}.19^{* * *} \\
\left(F_{14,169}=5.9\right)\end{array}$ & $\left(F_{5,169}^{.03^{*}}=2.5\right)$ & $\begin{array}{c}.11^{* * *} \\
\left(F_{10,169}=4.6\right)\end{array}$ \\
\hline Clutch size & Increase & $\begin{array}{c}.061^{* * *} \\
\left(F_{1,204}=15.7\right)\end{array}$ & $\left(F_{5,204}=1.3\right)$ & $\left(F_{14,204}^{.23^{* * *}}=5.8\right)$ & $\left(F_{5,204}^{.04^{*}}=2.9\right)$ & $\left(F_{11,204}^{.07^{*}}=2.3\right)$ \\
\hline $\begin{array}{l}\text { interciutch } \\
\text { interval }\end{array}$ & & $\left(F_{1,192}^{.00}=.3\right)$ & $\begin{array}{c}.08^{* * *} \\
\left(F_{5,192}=4.9\right)\end{array}$ & $\stackrel{.09}{\left(F_{17,192}=1.6\right)}$ & $\left(F_{5,192}^{.06^{* *}}=3.5\right)$ & $\left(F_{12,192}^{.10^{*}}=2.4\right)$ \\
\hline time & & $\begin{array}{c}.00 \\
\left(F_{1,159}=.1\right)\end{array}$ & $\begin{array}{c}.10^{* * *} \\
\left(F_{5,159}=5.4\right)\end{array}$ & $\stackrel{.08}{\left(F_{14,159}=1.5\right)}$ & $\begin{array}{c}.09^{* * *} \\
\left(F_{5,159}=4.6\right)\end{array}$ & $\left(F_{12,159}^{.15^{*}}=3.1\right)$ \\
\hline $\begin{array}{l}\text { Starvation } \\
\text { resistance }\end{array}$ & Increase & $\begin{array}{c}.15^{*} \\
\left(F_{1,27}=6.7\right)\end{array}$ & $\begin{array}{c}.15 \\
\left(F_{5,27}=1.4\right)\end{array}$ & NA & $\left(F_{5,27}^{.08}=.7\right)$ & NA \\
\hline
\end{tabular}

selecting for increased thermal tolerance for 10 generations and measuring the correlated selection response in six traits related to fitness, we could not detect any costs of thermal tolerance in selected lines compared to unselected controls. In fact, high thermal tolerance lines actually evolved increased body size, fecundity, and starvation resistance, suggesting a small benefit from (rather than costs of) increased thermal tolerance. These results imply that the plateau in the response to selection for increased thermal tolerance is due to a lack of additive genetic variation in the single trait (thermal tolerance) within populations rather than antagonistic selection imposed by negative pleiotropy or negative correlations with other traits under selection in the lab environment.

These results have important implications for adaptive responses to climate change in T. californicus. If alleles conferring greater thermal tolerance were present but failed to increase in frequency in our laboratory experiment due to stabilizing selection, then stronger selective forces (if present) should lead to the evolution of increased thermal tolerance in nature. However, if alleles conferring increased thermal tolerance are simply absent in natural populations, then this species must rely on new mutations or gene flow from lower-latitude populations for the evolution of increased thermal tolerance.
The absence of observed costs of increased thermal tolerance raises two related questions, discussed below.

1. Why is variation for thermal tolerance maintained within populations? Balancing selection imposed by tradeoffs is often invoked to explain the maintenance of genetic variation for tolerance traits in nature (Whittaker and Feeny 1971). Temperature and demographic data collected in the field for T. californicus both suggest that selection for increased thermal tolerance is common (fig. A2, available online). If increased tolerance incurs no cost, why have alleles conferring lower thermal tolerance persisted in nature? We see two possible answers. First, other traits correlated with thermal tolerance may be under-stabilizing selection in the field, thus maintaining genetic variation for thermal tolerance. For example, increased thermal tolerance was associated with the evolution of increased size, and size might in turn be under stabilizing selection. In general, it is important to note that the relative fitness of different genotypes is likely to be highly dependent on the context in which fitness is measured and that other unmeasured traits, such as disease resistance, might be important to fitness in the field.

Alternatively, variation for thermal tolerance may be maintained by antagonistic gene flow. Just as gene flow from the center of a species' geographic range may limit 

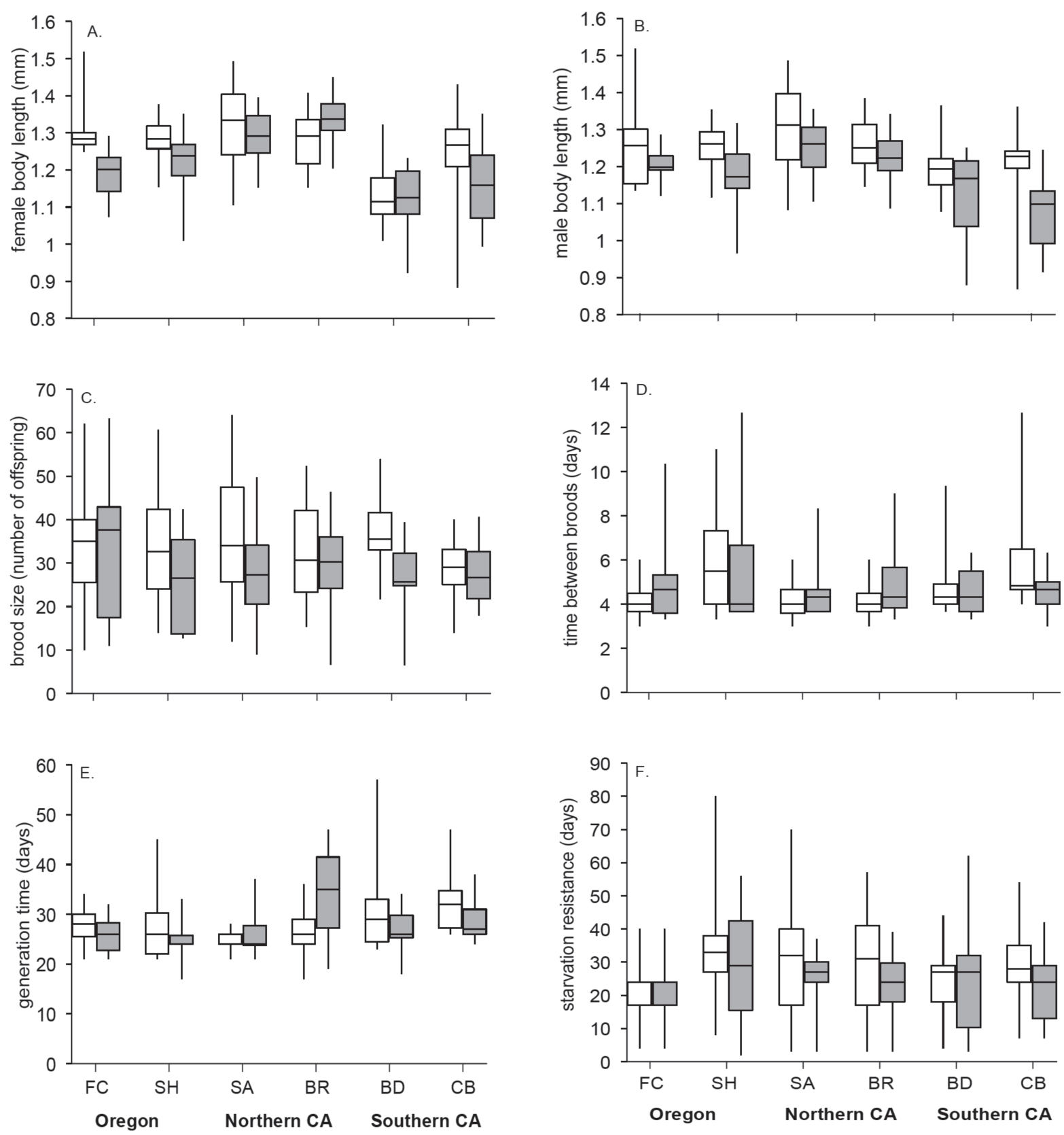

Figure 2: Box plots (quartiles) showing distribution of values of six traits-female size $(A)$, male size $(B)$, clutch size $(C)$, interclutch interval $(D)$, minimum generation time $(E)$, and starvation resistance (days survived without food; $F$ ) - for Tigriopus californicus from lines selected for thermal tolerance (white boxes) and unselected controls (gray boxes) from six populations.

the evolution of increased tolerance at range edges (Kirkpatrick and Barton 1997), gene flow from pools with more benign conditions could maintain thermal tolerance variation within sites. Antagonistic gene flow may limit niche expansion in a heterogeneous environment if habitat patches at the center of the niche have greater population size, density, or stability, so that net gene flow into mar- ginal parts of the habitat tends to prevent adaptation to conditions at the edge (Holt and Gaines 1992). This is possible even in the absence of a cost of increased tolerance, as in the case of mutational collapse of fitness in marginal habitats (Kawecki et al. 1997). Importantly, deeper and more thermally stable pools within a site also have larger and more persistent populations of T. califor- 
nicus, and these pools tend to serve as the source of new colonists when heating events cause local extinctions in shallower pools (M. W. Kelly, personal observation).

2. Why has the variation in thermal tolerance that is evident at the species level failed to evolve (or persist) in individual populations of T. californicus? Despite apparent selection for increased tolerance in the field (fig. A2) and lack of observed costs of increased thermal tolerance in the lab, northern populations of T. californicus have failed to evolve the higher tolerances found in southern populations of this species. One explanation for this incongruity is that the relationship between cost and tolerance is nonlinear, so that the cost imposed by thermal tolerance loci varies substantially with the size of the effect. Thus, the small amount of variation in thermal tolerance segregating within populations may be effectively neutral, whereas larger changes responsible for the differences between populations would incur a cost. We observed a maximum response to selection of $0.53^{\circ} \mathrm{C}$ within populations, whereas the differences in tolerance between the most and least tolerant populations were much larger at nearly $2^{\circ} \mathrm{C}$. Alternatively, the evolution of increased tolerance within populations may be limited primarily by drift and low gene flow. Populations of T. californicus are highly subdivided, and so beneficial alleles that arise in some populations may not spread to others due to low migration rates (Burton and Lee 1994; Edmands 2001).

Our inability to detect a cost of thermal tolerance contrasts with the findings of Willett (2010), who found a trade-off in performance at $16^{\circ} \mathrm{C}$ versus $24^{\circ} \mathrm{C}$ in $T$. californicus. However, our experiments measured the cost of tolerance variation segregating within populations, whereas Willett (2010) measured variation in performance among populations. Furthermore, we measured costs of increased upper lethal temperatures, whereas Willett (2010) measured variation in performance at different ambient temperatures. Selection experiments in Drosophila have demonstrated that these two measures of tolerance do not necessarily have the same genetic basis (Hoffmann et al. 2003b; Bubliy and Loeschcke 2005).

\section{Geographic Variation in the $\mathbf{G}$ Matrix}

Notably, the correlated effects of selection for increased thermal tolerance on other traits varied by population for all measured traits except male size. This strongly suggests that the genetic basis for the response to selection varied among geographically separated populations. This is perhaps unsurprising, given the high level of differentiation among populations of T. californicus at neutral loci (Edmands 2001; Kelly et al. 2012) and evidence that drift and bottlenecks can lead to changes in genetic correlations among traits in other species (Shaw et al. 1995; Whitlock et al. 2002).

Genetic correlations among traits were once thought to be constant enough to allow retrospective inference of the selection pressures leading to divergence among closely related species (Lande 1979). It is now generally accepted that the conditions necessary for the constancy of the $\mathbf{G}$ matrix are unlikely to be met by real genomes and populations (Turelli 1988; Shaw et al. 1995). Our work adds to the small but growing body of evidence that the G matrix may vary not only among closely related species but also among geographically separated populations of the same species (Cohan and Hoffmann 1986, 1989; Roff et al. 2004). This leads to variation among populations in the correlated responses to selection and, in some cases (e.g., female body size in our data; fig. $2 A$ ), even to correlated responses that are in the opposite direction for populations separated by $<200 \mathrm{~km}$.

Evolutionary change has the power to influence ecological processes, and the capacity to adapt to changing conditions may mitigate extinction risk for natural populations (Gomulkiewicz and Holt 1995; Bell and Gonzalez 2009). However, the capacity of most populations to evolve in response to a changing environment is poorly understood. Key to this understanding is a full description of the magnitude of genetic variation for environmental tolerance and the relationships between environmental tolerance and other traits. Our results show that these relationships can be complicated: correlations among traits differ among populations of T. californicus, and while genetic variation for thermal tolerance within populations is extremely limited in comparison to the species as a whole, we found no evidence that this limitation stems from fitness trade-offs between thermal tolerance and other traits within populations.

\section{Acknowledgments}

We thank D. Grossenbacher, M. Turelli, J. Wright, and two anonymous reviewers for helpful comments on an earlier version of this manuscript. Laboratory assistance was provided by F. Liu, J. Louie, M. Nguyen, K. Sato, B. Sayedi, J. Tang, and I. Wong, and we thank B. Cameron for logistical advice. This work was funded by National Science Foundation grants DDIG 09-09788, OCE-06-22924, and OCE-09-29057 to M.W.K., E.S., and R.K.G., respectively.

\section{Literature Cited}

Agrawal, A. A., J. K. Conner, and S. Rasmann. 2010. Tradeoffs and negative correlations in evolutionary ecology. Pages 243-268 in M. 
A. Bell, D. J. Futuyma, W. F. Eanes, and J. S. Levinton, eds. Evolution since Darwin: the first 150 years. Sinauer, Sunderland, MA. Agrawal, A. F., and J. R. Stinchcombe. 2009. How much do genetic covariances alter the rate of adaptation? Proceedings of the Royal Society B: Biological Sciences 276:1183-1191.

Armbruster, W. S., and K. E. Schwaegerle. 1996. Causes of covariation of phenotypic traits among populations. Journal of Evolutionary Biology 9:261-276.

Balanya, J., J. M. Oller, R. B. Huey, G. W. Gilchrist, and L. Serra. 2006. Global genetic change tracks global climate warming in Drosophila subobscura. Science 313:1773-1775.

Barton, N. H., and M. Turelli. 1989. Evolutionary quantitative genetics: how little do we know? Annual Review of Genetics 23:337370 .

Bell, G., and A. Gonzalez. 2009. Evolutionary rescue can prevent extinction following environmental change. Ecology Letters 12: 942-948.

Blows, M. W., and A. A. Hoffmann. 2005. A reassessment of genetic limits to evolutionary change. Ecology 86:1371-1384.

Brakefield, P. M. 2003. Artificial selection and the development of ecologically relevant phenotypes. Ecology 84:1661-1671.

Bubliy, O. A., and V. Loeschcke. 2005. Correlated responses to selection for stress resistance and longevity in a laboratory population of Drosophila melanogaster. Journal of Evolutionary Biology 18:789-803.

Burton, R. S., and B. N. Lee. 1994. Nuclear and mitochondrial gene genealogies and allozyme polymorphism across a major phylogeographic break in the copepod Tigriopus californicus. Proceedings of the National Academy of Sciences of the USA 91:5197-5201.

Cano, J. M., A. Laurila, J. Palo, and J. Merilä. 2004. Population differentiation in $\mathbf{G}$ matrix structure due to natural selection in Rana temporaria. Evolution 58:2013-2020.

Carriere, Y., J. P. Deland, D. A. Roff, and C. Vincent. 1994. Lifehistory costs associated with the evolution of insecticide resistance. Proceedings of the Royal Society B: Biological Sciences 258:35-40.

Charlesworth, B. 1990. Optimization models, quantitative genetics, and mutation. Evolution 44:520-538.

Chown, S. L., and K. J. Gaston. 2008. Macrophysiology for a changing world. Proceedings of the Royal Society B: Biological Sciences 275: 1469-1478.

Cohan, F. M., and A. A. Hoffmann. 1986. Genetic divergence under uniform selection. II. Different responses to selection for knockdown resistance to ethanol among Drosophila melanogaster populations and their replicate lines. Genetics 114:145-164.

- 1989. Uniform selection as a diversifying force in evolution: evidence from Drosophila. American Naturalist 134:613-637.

Conner, J. K., K. Karoly, C. Stewart, V. A. Koelling, H. F. Sahli, and F. H. Shaw. 2011. Rapid independent trait evolution despite a strong pleiotropic genetic correlation. American Naturalist 178: 429-441.

Doroszuk, A., M. W. Wojewodzic, G. Gort, and J. E. Kammenga. 2008. Rapid divergence of genetic variance-covariance matrix within a natural population. American Naturalist 171:291-304.

Edmands, S. 2001. Phylogeography of the intertidal copepod Tigriopus californicus reveals substantially reduced population differentiation at northern latitudes. Molecular Ecology 10:1743-1750.

Etterson, J. R., and R. G. Shaw. 2001. Constraint to adaptive evolution in response to global warming. Science 294:151-154.

Franks, S. J., and A. A. Hoffmann. 2012. Genetics of climate change adaptation. Annual Review of Genetics 46:185-208.
Franks, S. J., S. Sim, and A. E. Weis. 2007. Rapid evolution of flowering time by an annual plant in response to a climate fluctuation. Proceedings of the National Academy of Sciences of the USA 104: $1278-1282$.

Fry, J. D. 2003. Detecting ecological trade-offs using selection experiments. Ecology 84:1672-1678.

Gilchrist, G, W., R. B. Huey, and L. Partridge. 1997. Thermal sensitivity of Drosophila melanogaster: evolutionary responses of adults and eggs to laboratory natural selection at different temperatures. Physiological Zoology 70:403-414.

Gomulkiewicz, R., and R. D. Holt. 1995. When does evolution by natural selection prevent extinction? Evolution 49:201-207.

Gromko, M. H. 1995. Unpredictability of correlated response to selection: pleiotropy and sampling interact. Evolution 49:685-693.

Hoffmann, A. A., A. Anderson, and R. Hallas. 2002. Opposing clines for high and low temperature resistance in Drosophila melanogaster. Ecology Letters 5:614-618.

Hoffmann, A. A., H. Dagher, M. Hercus, and D. Berrigan. 1997. Comparing different measures of heat resistance in selected lines of Drosophila melanogaster. Journal of Insect Physiology 43:393405.

Hoffmann, A. A., R. J. Hallas, J. A. Dean, and M. Schiffer. $2003 a$. Low potential for climatic stress adaptation in a rainforest Drosophila species. Science 301:100-102.

Hoffmann, A. A., and C. M. Sgrò. 2011. Climate change and evolutionary adaptation. Nature 470:479-485.

Hoffmann, A. A., J. G. Sørensen, and V. Loeschcke. 2003b. Adaptation of Drosophila to temperature extremes: bringing together quantitative and molecular approaches. Journal of Thermal Biology 28: 175-216.

Holt, R. D., and M. S. Gaines. 1992. Analysis of adaptation in heterogeneous landscapes: implications for the evolution of fundamental niches. Evolutionary Ecology 6:433-447.

Houle, D. 1991. Genetic covariance of fitness correlates: what genetic correlations are made of and why it matters. Evolution 45:630648.

Huey, R. B., and J. G. Kingsolver. 1993. Evolution of resistance to high temperature in ectotherms. American Naturalist 142(suppl.): S21-S46.

Janmaat, A. F., and J. H. Myers. 2005. The cost of resistance to Bacillus thuringiensis varies with the host plant of Trichoplusia ni. Proceedings of the Royal Society B: Biological Sciences 272:1031-1038.

Jones, A. G., S. J. Arnold, and R. Bürger. 2003. Stability of the Gmatrix in a population experiencing pleiotropic mutation, stabilizing selection, and genetic drift. Evolution 57:1747-1760.

Jump, A. S., and J. Peñuelas. 2005. Running to stand still: adaptation and the response of plants to rapid climate change. Ecology Letters 8:1010-1020.

Kawecki, T. J., N. H. Barton, and J. D. Fry. 1997. Mutational collapse of fitness in marginal habitats and the evolution of ecological specialisation. Journal of Evolutionary Biology 10:407-429.

Kelly, M. W., E. Sanford, and R. K. Grosberg. 2012. Limited potential for adaptation to climate change in a broadly distributed marine crustacean. Proceedings of the Royal Society B: Biological Sciences 279:349-356.

Kirkpatrick, M., and N. H. Barton. 1997. Evolution of a species' range. American Naturalist 150:1-23.

Lande, R. 1979. Quantitative genetic analysis of multivariate evolution, applied to brain : body size allometry. Evolution 33:402416. 
Lande, R., and S. J. Arnold. 1983. The measurement of selection on correlated characters. Evolution 37:1210-1226.

Loeschcke, V., and R. A. Krebs. 1996. Selection for heat-shock resistance in larval and in adult Drosophila buzzatii: comparing direct and indirect responses. Evolution 50:2354-2359.

Lohbeck, K. T., U. Riebesell, and T. B. H. Reusch. 2012. Adaptive evolution of a key phytoplankton species to ocean acidification. Nature Geoscience 5:346-351.

Mauricio, R., and M. D. Rausher. 1997. Experimental manipulation of putative selective agents provides evidence for the role of natural enemies in the evolution of plant defense. Evolution 51:1435-1444.

Mongold, J. A., A. F. Bennett, and R. E. Lenski. 1999. Evolutionary adaptation to temperature. VII. Extension of the upper thermal limit of Escherichia coli. Evolution 53:386-394.

Palaima, A., and K. Spitze. 2004. Is a jack-of-all-temperatures a master of none? an experimental test with Daphnia pulicaria (Crustacea: Cladocera). Evolutionary Ecology Research 6:215-225.

R Development Core Team. 2010. R: a language and environment for statistical computing. R Foundation for Statistical Computing, Vienna.

Robertson, A. 1960. A theory of limits in artificial selection. Proceedings of the Royal Society B: Biological Sciences 153:234-249.

Roff, D. A., T. Mousseau, A. P. Moller, F. De Lope, and N. Saino. 2004. Geographic variation in the $\mathbf{G}$ matrices of wild populations of the barn swallow. Heredity 93:8-14.

Shaw, F. H., R. G. Shaw, G. S. Wilkinson, and M. Turelli. 1995. Changes in genetic variances and covariances: $G$ whiz! Evolution 49:1260-1267.

Shine, R. 1988. The evolution of large body size in females: a critique of Darwin's "fecundity advantage" model. American Naturalist 131:124-131.
Spitze, K. 1993. Population structure in Daphnia obtusa: quantitative genetic and allozymic variation. Genetics 2:367-374.

Stebbins, G. L. 1950. Variation and evolution in plants. Columbia University Press, New York.

Turelli, M. 1988. Phenotypic evolution, constant covariances and the maintenance of additive variance. Evolution 42:1342-1347.

Weber, K. E., and L. T. Diggins. 1990. Increased selection response in larger populations. 2. Selection for ethanol vapor resistance in Drosophila melanogaster at two population sizes. Genetics 125:585597.

Whitlock, M. C., P. C. Phillips, and K. Fowler. 2002. Persistence of changes in the genetic covariance matrix after a bottleneck. Evolution 56:1968-1975.

Whittaker, R. H., and P. P. Feeny. 1971. Allelochemics: chemical interactions between species. Science 171:757-770.

Willett, C. S. 2010. Potential fitness trade-offs for thermal tolerance in the intertidal copepod Tigriopus californicus. Evolution 64:25212534.

Williams, B. R., B. van Heerwaarden, D. K. Dowling, and C. M. Sgrò. 2012. A multivariate test of evolutionary constraints for thermal tolerance in Drosophila melanogaster. Journal of Evolutionary Biology 25:1415-1426.

Worley, A. C., and S. C. H. Barrett. 2000. Evolution of floral display in Eichhornia paniculata (Pontederiaceae): direct and correlated responses to selection on flower size and number. Evolution 54: 1533-1545.

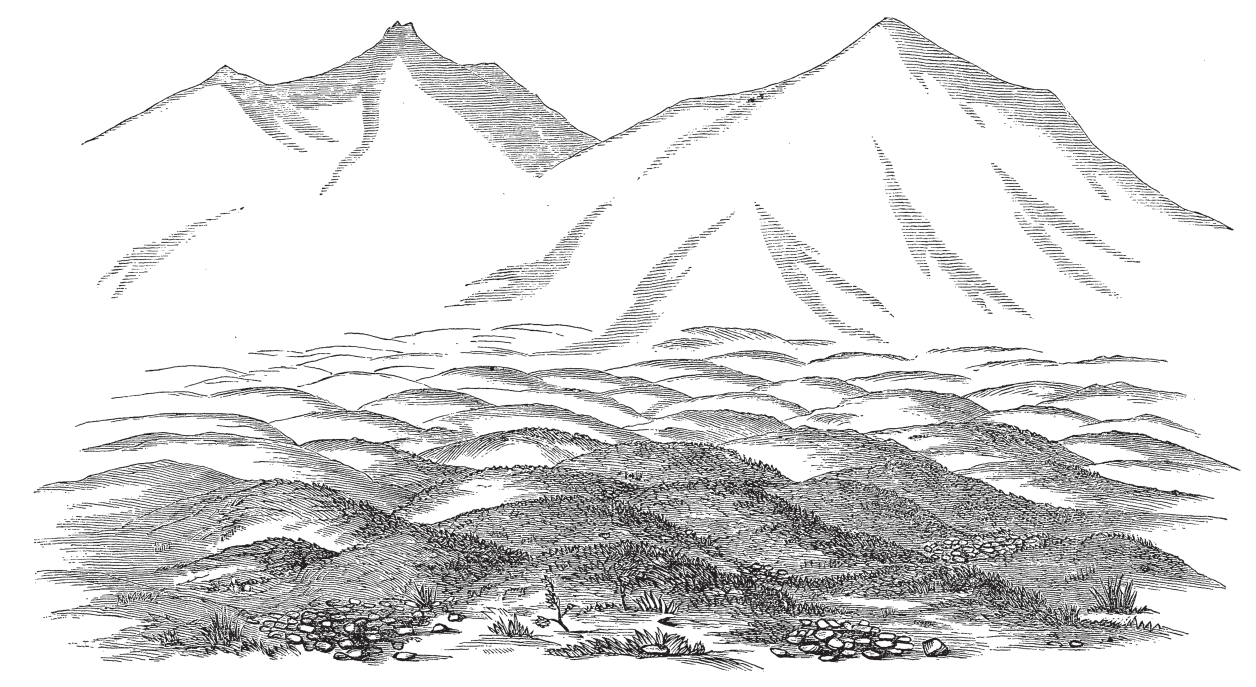

\footnotetext{
"The surface geology of many sections of the Pacific slope is characterized by innumerable hillocks or small mound-like formations, either sparsely distributed or occupying quite densely areas of considerable extent. These formations, variable in size and structure in accordance with local conditions concerned in their production, exist in many parts of California and on the coast north of it, and are especially abundant and well defined in Southern California." From "The Hillocks or Mound-Formations of San Diego, California” by G. W. Barnes (American Naturalist, 1879, 13:565-571).
} 